\title{
Mars Atmosphere and Volatile Evolution Mission Instrument Integration and Test Challenges
}

\author{
Nicholas Jedrich \\ NASA Goddard Space Flight Center \\ 8800 Greenbelt Road \\ Greenbelt, MD 20770 \\ 301-286-6367 \\ Nicholas.M.Jedrich@nasa.gov
}

\author{
Regan Howard \\ Orbital Sciences Corporation \\ 7500 Greenbelt Ctr Dr, Suite 700 \\ Greenbelt, MD 20771 \\ 301-286-4450 \\ howard.regan@orbital.com
}

\begin{abstract}
This paper focuses on the many challenges involved in the integration and test of the Mars Atmosphere and Volatile EvolutioN (MAVEN) mission instrument packages both standalone and with the spacecraft. MAVEN is on schedule for a November 2013 launch from Kennedy Space Center (KSC) in Florida. The instrument packages manifested for MAVEN will provide the data required to determine the impact on climate due to atmospheric loss to space. MAVEN will accomplish this by measuring the structure, composition, and variability of all regions from which escape occurs, and will sample all local solar times and most latitudes. The requirements imposed on the mission dictate the need for a rigorous ground test program and well defined spacecraft interfaces, while the fixed launch date requires innovative and timely solutions to reduce both technical and schedule risk during the test program. The test limitations and constraints will be covered, with unique tests and methods discussed in detail. Among these are high voltage operations, magnetic and optics testing. The mechanical and electrical interfaces to the spacecraft, along with the instrument interactions that drove those interface requirements will be included. All the MAVEN instruments have been successfully integrated to the spacecraft; and lessons learned that have resulted in a test program that not only lowers risk, but allowed the majority of the instruments to adhere to their original delivery schedules will be presented.
\end{abstract}

\section{TABLE OF CONTENTS}

1. INTRODUCTION ............................................1

2. INSTRUMENT TO SPACECRAFT INTERFACES....2

3. Test Program .6

4. CONCLUSIONS ....................................................9

BIOGRAPHY ....................................................10

ACKNOWLEDGEMENTS.................................10

\section{INTRODUCTION}

Mission and Science

The MAVEN mission is a Principal Investigator (PI) led mission with the NASA Goddard Space Flight Center (GSFC) responsible for technical implementation and management. It was launched from Cape Canaveral Air Force Station (CCAFS) on November 18, 2013, with Mars Orbit Insertion (MOI) to occur on September 22, 2014, followed by a primary science mission of one Earth year in duration.

For the past decade, the possible presence of liquid water on Mars, either in its ancient past or preserved in the subsurface today led to the development of an exploration strategy generally known as "Follow the Water". Abundant evidence for ancient liquid water on Mars, as shown in Fig. 1, but not today, suggests dramatic climate change.

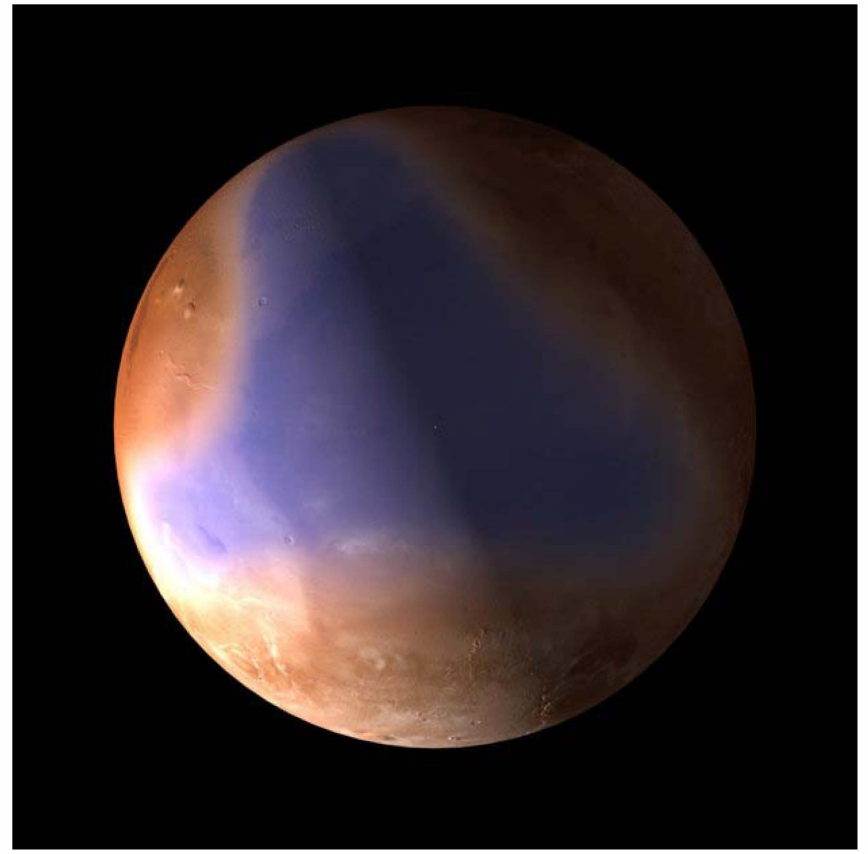

Figure 1 - ESA Mars Express: Results from the MARSIS (Mars Advanced Radar for Subsurface and Ionospheric Sounding) radar on Mars Express give strong evidence for a former ocean of Mars

Evidence increasingly points to atmospheric loss to space as being a major driver of this change. MAVEN's investigation of the present state of the upper atmosphere and ionosphere, the processes controlling them and the determination of how loss of volatiles to outer space in the present epoch varies with changing solar conditions, is designed to provide definitive answers about Mars climate history. 


\section{Instruments and Science}

Under the premise that atmospheric loss to space is the major driver of atmospheric change, instruments that collect in situ and mapping data are essential to understanding the processes involved.

The MAVEN instrument selection consists of: standalone Neutral Gas and Ion Mass Spectrometer (NGIMS); an Imaging UltraViolet Spectrometer (IUVS) referred to as the
Remote Sensing (RS) Package; and the Particles and Fields Package (PFP) composed of six instruments: Solar Wind Ion Analyzer (SWIA), Solar Wind Electron Analyzer (SWEA), Langmuir Probe and Waves (LPW)/Extreme UltraViolet (EUV), Magnetometer (MAG), Solar Energetic Particle (SEP), and SupraThermal And Thermal Ion Composition (STATIC). The instrument locations are shown in Fig. 2.

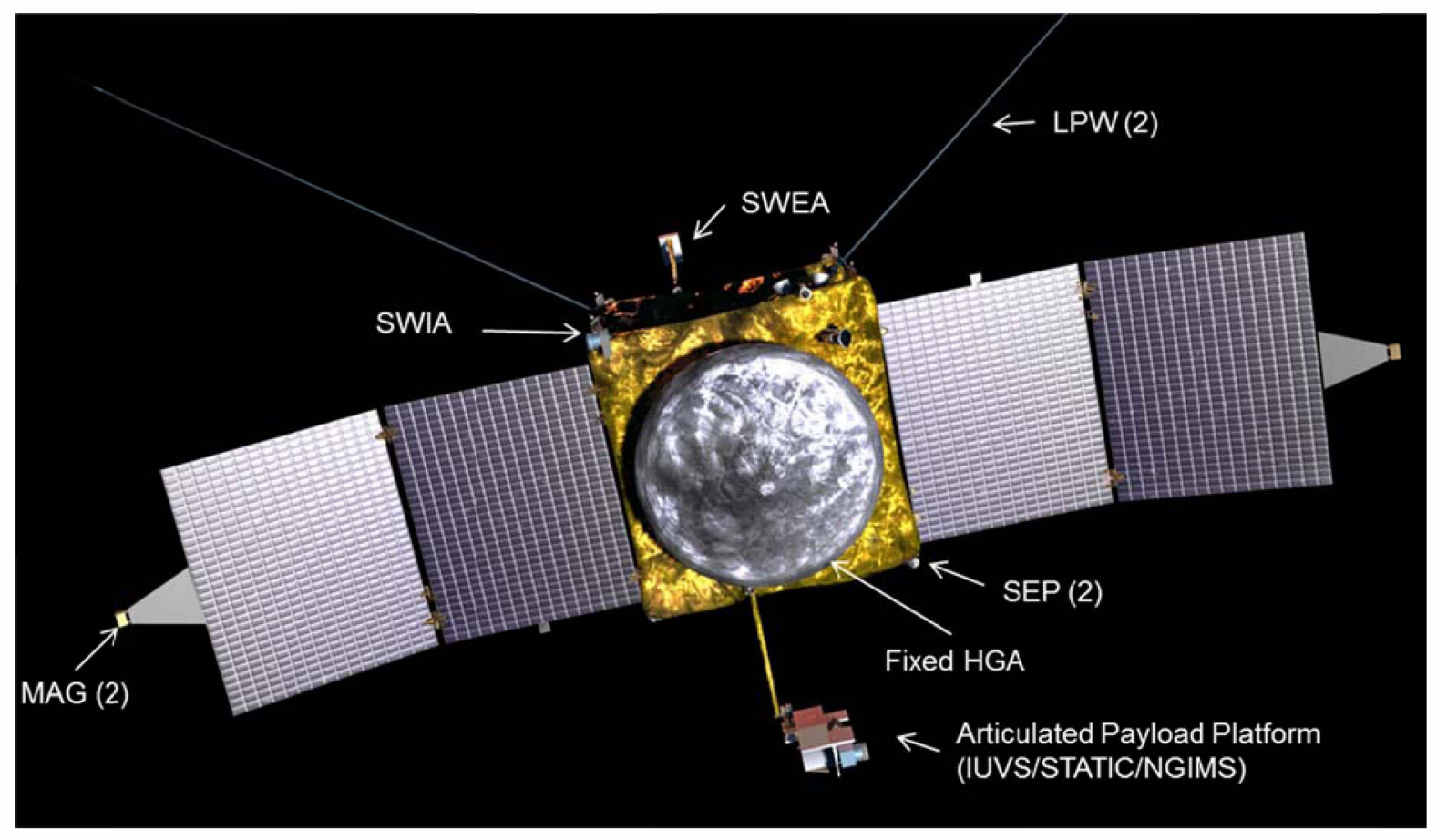

Figure 2 - Instruments are mounted to the Spacecraft, Solar Array (SA) and the gimbaled Articulating Payload Platform (APP)

In situ measurements from NGIMS focus on the structure and composition of the upper atmosphere and ionosphere, while related data mapped over the planet is provided by IUVS. The PFP instruments measure: ionospheric properties, energetic ions and electrons, solar wind and solar energetic particles, magnetic fields, and solar EUV irradiance. Combining the instrument data with empirical models allows extrapolation of total atmospheric loss to earlier epochs, reference Fig. 3. These data contribute to better understanding of all of the atmospheric loss and energy processes, and how important the escape to space has been in the history of Mars' climate.

\section{INSTRUMENT TO SPACECRAFT INTERFACES}

MAVEN mission requirements drove unique instrument to spacecraft interfaces both mechanically and electrically. These requirements were defined thoroughly via collaboration between the instrument and spacecraft provider, and agreed upon early during the project lifecycle. As a result, cost and schedule risk was mitigated. The following sections will cover the most challenging of the instrument interfaces and the design solutions that enabled their requirements to be met. Sections 3 will provide insight into how some of those same requirements were verified during the test program.

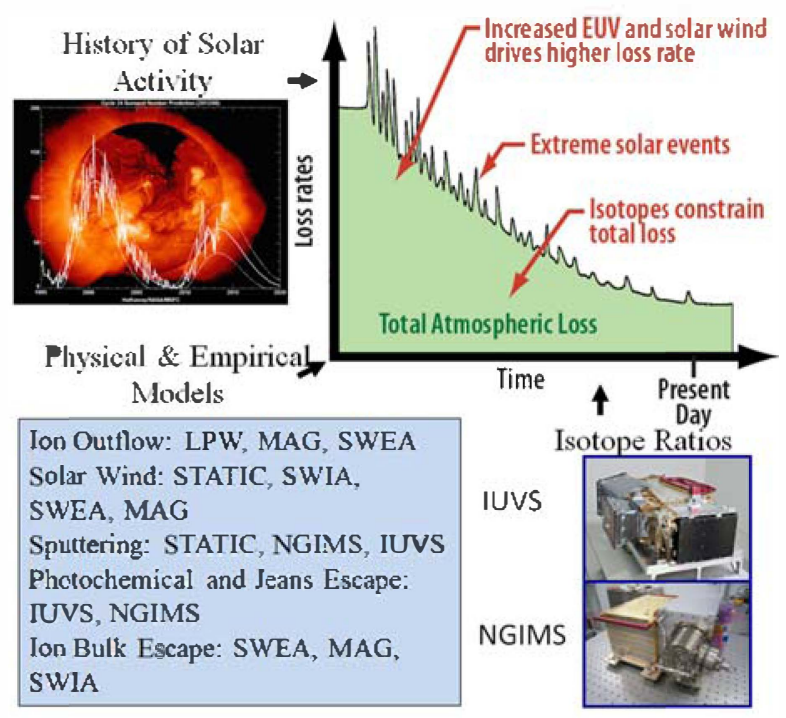

Figure 3 - Approach to Determine Atmospheric Loss 


\section{Magnetometer to Solar Array}

Requirements - Two magnetometers measure the magnitude and direction of the various magnetic fields including: interplanetary, induced magnetosphere, ionosphere, and crustal. They provide a crucial role in understanding Mars' plasma environment as they provide the magnetic field context for the measurements from SWEA, SWIA and STATIC plasma instruments. The measurement requirements are driven by the relatively weak field environment of the solar wind and the more intense magnetic fields produced by crustal magnetic anomalies near the Martian surface ${ }^{1}$. MAG must support a dynamic range of 3 to $3000 \mathrm{nT}$ covering from the very weak (1 to 2 nT) fields of the Interplanetary Magnetic Field (IMF), the merely weak (10 to $50 \mathrm{nT}$ ) fields due to the solar wind interaction with Mars' plasmasphere, and finally to the relatively strong (100 to $1000 \mathrm{nT}$ ) fields in the crusts of the Southern Highlands. The use of two magnetometers allows for a differential measurement mode providing greater measurement sensitivity as well as partial redundancy should one magnetometer fail.

The requirement to measure very weak fields with adequate precision placed severe limits on what the magnetometers were allowed to see from the spacecraft. Level 2 (L2) requirements were imposed for the dynamic "Alternating Current" (AC) and static "Direct Current" (DC) requirements respectively:

- The AC magnetic field produced by the orbiter, and seen at each magnetometer, shall be less than $0.25 \mathrm{nT}$ from 0 to $10 \mathrm{~Hz}$, except during transitions in/out of eclipse and in/out of off-sun pointing modes.

- The DC magnetic field produced by the orbiter, and seen at each magnetometer, shall be less than $2 \mathrm{nT}$.

Two Level 3 (L3) requirements specify implementation as follows:

- No magnetic materials shall be used within 26.8" (.68 $\mathrm{m})$ of the MAG sensors in the deployed SA configuration.

- Mounting brackets for MAG1 and MAG2 will be provided by GSFC to mount the MAG instrument to the spacecraft.

Design Solution - As in many missions with magnetometers, the first objective for accommodating a science magnetometer on a spacecraft is to keep it as far from the spacecraft generated fields as possible. Seven options to keep the MAG sensors away from the spacecraft bus were traded including modification of the SWEA boom for mounting both MAGs in addition to SWEA on a single boom, separate deployable booms for each MAG extending from the spacecraft structure, and two extended hinged segments attached to the end of each SA to which a MAG would be mounted.
Most of the trades resulted in unwarranted technical complexities, increased risk, and significant cost impacts. The trade selected took advantage of the existing solar array panel substrate length to mount the sensors on extended "boomlets" located at the end of each solar array, reference Fig. 4. This solution results in $0.68 \mathrm{~m}$ of separation between the closest edge of the solar array and the sensor. The use of a composite bracket with non-magnetic fasteners, reference Fig. 5, completes the implementation.

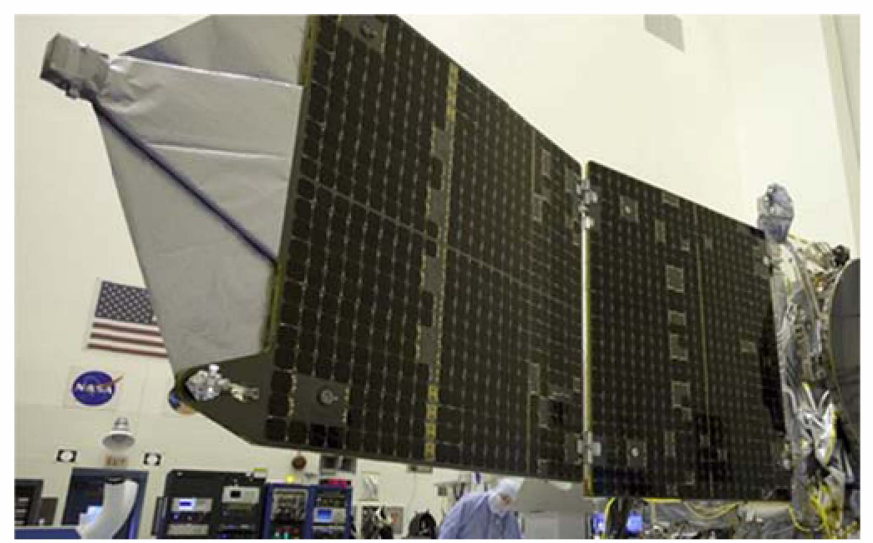

Figure 4-MAG mounted to Solar Array for Flight

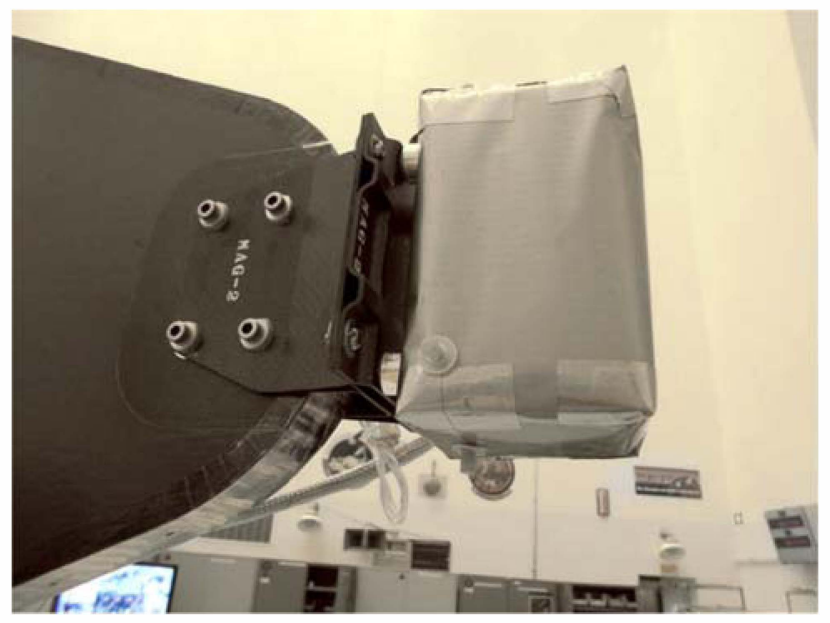

Figure 5 - MAG mounted to its bracket attached to the end of the "boomlet" on the SA

The selected approach effectively placed the magnetometers on the equivalent of $\sim 6.0 \mathrm{~m}$ booms when measured from the center of the spacecraft. This spatial separation from majority of the spacecraft magnetic field sources greatly reduced the effort needed to meet the L2 static and dynamic spacecraft magnetic cleanliness requirements. The proximity to the solar array strings, however, led to the need to design the solar array panels to be magnetically clean at the location of the magnetometers. Unlike the Mars Global Surveyor spacecraft, MAVEN was able to use the backside of the solar array panels for string backwiring. This permitted the use of a self-canceling string configuration (Fig. 6), similar to those used on the Juno and Radiation Belt Storm Probe missions, which when combined with a "checkerboard" string polarity layout, resulted in field magnitudes of $<0.1 \mathrm{nT}$ at the magnetometers. 


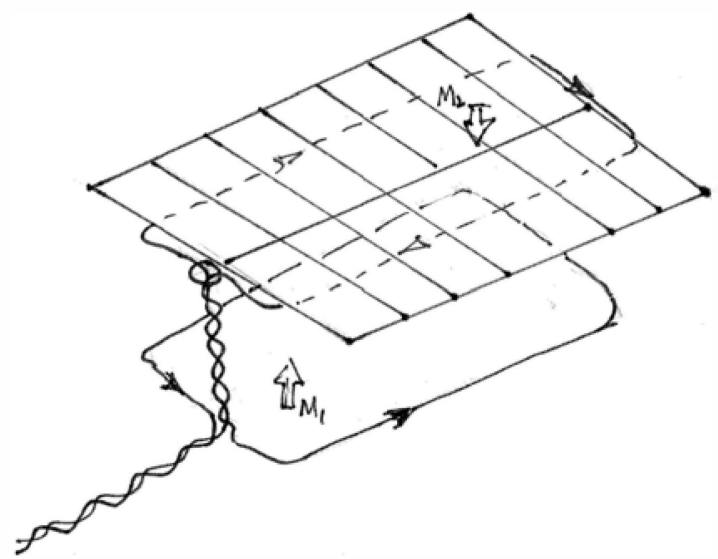

Figure 6 - Self-Canceling Solar Array String
Configuration

During the design efforts models were maintained of both the static and dynamics fields expected at the magnetometers. The models gave the spacecraft team the insight necessary to make effective trades and also the measurement framework against which spacecraft level testing was made. Both static and dynamics models indicated that the MAVEN magnetic cleanliness requirements were being met.

\section{Instrument to Spacecraft Body and APP}

Requirements - The instrument complement on MAVEN was designed to capture the processes driving the volatile loss to space by simultaneously looking at the forcing mechanism, solar influences, and the resulting structure and composition of the upper atmosphere and ionosphere and escaping ions. Not unlike other missions with multiple instruments making multiple measurements, MAVEN requires Fields of View (FOVs) that are somewhat in conflict with each other. Note: LPW will be covered in the next section as it has some very unique requirements.

The L3 Interface Control Documents (ICDs) contain the following with respect to instrument FOVs:

- The spacecraft shall provide the FOVs, including infringements, as shown in the Mechanical Interface Control Document (MICD).

- SWIA shall be mounted such that its 360 by 100 degrees FOV is in the $\mathrm{x}-\mathrm{z}$ plane and oriented such that a $+/-30$ degree by +/-30 degree region within the FOV parallel to the spacecraft Z-axis is unobstructed (nominally sun-pointed).

- The two SEP sensors shall be mounted such that each boresight has a glint-free FOV (completely unobstructed) of 50 by 41 degrees with conical radiused corners. The SEP sensors will be oriented to provide maximum coverage of the Parker spiral field direction as agreed to by the payload team.
- EUV shall be mounted such that each boresight has a 24 degree full cone ( \pm 12 degree half cone) glint-free FOV parallel to the Z-axis (nominally sun-pointed).

- The spacecraft shall mount the IUVS instrument to nominally keep sun out of its FOV to avoid permanent damage to the sensor.

- SWEA shall be mounted such that its 360 by 130 degrees FOV is in the $x-y$ plane and does not include the Sun when the spacecraft is in normal sun-pointing mode. Spacecraft obstructions will be minimized as agreed to by the payload team.

- STATIC shall be mounted on the APP with its 360 by 90 degrees FOV in the $i-j$ plane and oriented such that a $+/-45$ degree region within the FOV around the $\mathrm{i}$-axis is locally unobstructed. Spacecraft obstructions will be minimized as agreed to by the payload team.

- NGIMS shall be mounted on the APP such that its 16 degree conical open source FOV is centered parallel with the APP i-axis with 20 degree unobstructed and the 2 pi-steridian closed source FOV centered parallel to the APP i-axis is locally unobstructed and the 2 pisteridian vent FOV centered parallel to the APP $-\mathrm{k}$ axis is locally unobstructed.

Design Solution - The design solution for solar facing FOV instruments is straight forward, they are positioned on the top deck of the spacecraft with unobstructed views towards the sun in most observing modes. This is the case for the EUV (reference Fig. 7), and SWIA instruments. In addition, the two SEP instruments are mounted on the same spacecraft deck to keep the sun out of the sensor and provide the best sampling of solar wind electrons, Fig. 8.

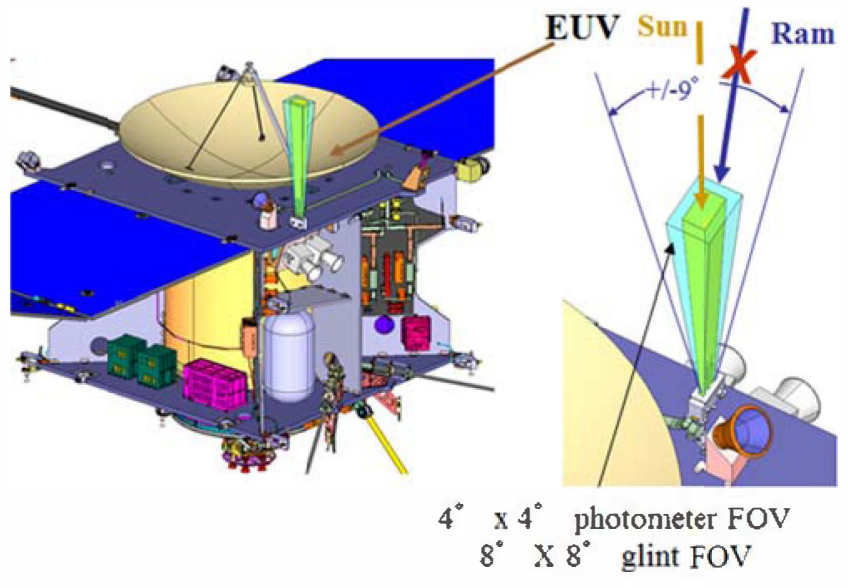

Figure 7 - EUV FOV 


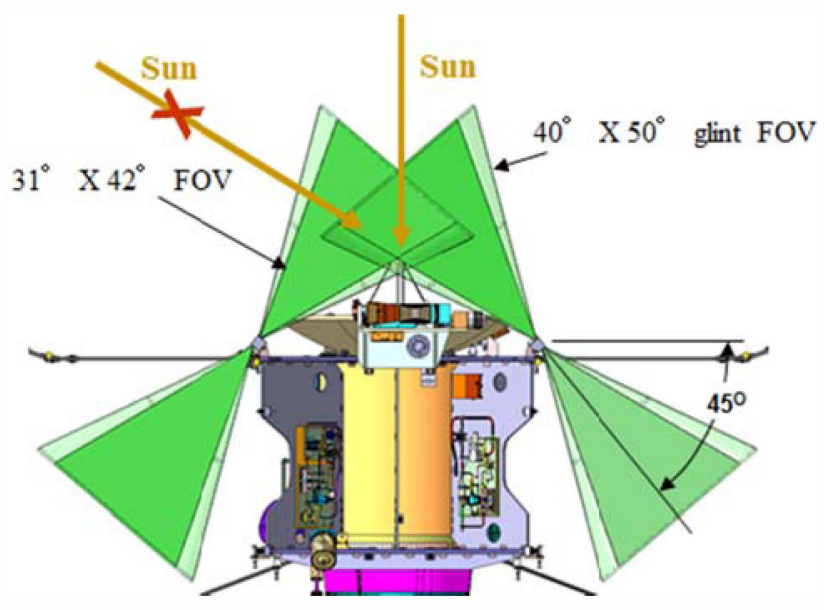

Figure 8 - SEP FOVs

Four of the MAVEN instruments are mounted on booms, one on its own boom and three on the APP respectively, reference Fig. 2. SWEA is placed on the end of a $1.65 \mathrm{~m}$ boom, which allows a large, clear FOV and keeps the trajectories of electrons travelling to SWEA as far as possible from the spacecraft ${ }^{2}$. The IUVS, STATIC, and NGIMS instruments are on the APP, a two axis gimbal platform, allowing the instruments to be pointed independent of the spacecraft body.

\section{LPW to Spacecraft}

Requirements - Two LPWs are required to determine thermal electron density (100 to $\left.10^{6} \mathrm{~cm}^{-3}\right)$ and electron temperature $(500-5000 \mathrm{~K})$, below the ionopause, with density precision of $20 \%$ and relative precision of at least $5 \%$ and temperature precision of at least $20 \%$ with a vertical resolution better than $60 \mathrm{~km}$. This will provide profiles of electron number density and temperature, and provide important constraints on ionospheric, photochemical, and solar-wind-related processes. In addition, LPW determines electric field waves at frequencies between 0.05 and $10 \mathrm{~Hz}$ at altitudes from $200 \mathrm{~km}$ (nominal exobase) to $600 \mathrm{~km}$ (above the nominal ionopause). Wave power sensitivity of at least $1 \times 10^{8}(\mathrm{~V} / \mathrm{m})^{2} / \mathrm{Hz}$ is required.

The requirement to measure very weak quasi-static electric fields and waves with adequate precision placed severe limits on what the Langmuir Probe sensors were allowed to see from the spacecraft. L2 requirements were imposed for the both the potential variation over the spacecraft surface, ratio of conductive grounded area to biased metal area, and the power density allowed in the frequency band of operation. In particular:

- The maximum tolerable variation in potential across the surface of the Orbiter, dVmax, shall be 1 Volt, with a goal of 0.1 Volts.

- The ratio of grounded, conductive current collecting area on the Orbiter to the area of all surfaces at a biased potential must exceed 200.
- The solar array string switching shall not generate string switching RF emissions in the LPW spectrum for a contiguous block of at least $50 \%$ of each 1 second solar array string switching cycle as follows:

\begin{tabular}{|c|c|c|}
\hline Driver & Frequency Range & Radiated Electric Field Limit \\
\hline $\mathrm{LPW}$ & $0.05-10 \mathrm{~Hz}$ & 85 to $40 \mathrm{dBuV} / \mathrm{m}$ (by analysis only) \\
\hline $\mathrm{LPW}$ & $14 \mathrm{kHz}-4 \mathrm{MHz}$ & $60 \mathrm{dBuV} / \mathrm{m}$ \\
\hline
\end{tabular}

- The MAVEN orbiter shall control low frequency noise coupling to the Langmuir probe instrument to the levels specified below:

\begin{tabular}{|l|l|}
\hline \multicolumn{1}{|c|}{ Frequency Range } & \multicolumn{1}{c|}{ Radiated Electric Field Limit } \\
\hline $100 \mathrm{~Hz}$ to $14 \mathrm{kHz}$ & $60 \mathrm{dBuV} / \mathrm{m}$ \\
\hline $14 \mathrm{kHz}-2 \mathrm{MHz}$ & 45 to $35 \mathrm{dBuV} / \mathrm{m}$ \\
\hline$>2 \mathrm{MHz}$ & $60 \mathrm{dBuV} / \mathrm{m}$ \\
\hline
\end{tabular}

Spacecraft accommodation of LPW was dictated by the three L2 requirements listed above plus the L3 LPW ICD which contained the following with respect to instrument FOVs:

- The two LPW sensors shall be oriented such that at least one is outside the spacecraft wake and shadow at all times.

- The tip of each LPW sensor shall be at least $5 \mathrm{~m}$ from the closest part of the spacecraft (including solar arrays and other appendages).

- The deployed LPW and boom assembly shall have a minimum first mode natural frequency $>/-0.25 \mathrm{~Hz}$.

An additional requirement for Integration and Test reasons is:

- The LPW whips shall be removable.

Design Solution - The variation in potential requirement was satisfied for the most part using conductive thermal blankets; however, an exception was made for the solar cell surfaces where a conductive coating was not used for cost reasons. Detailed analysis showed this exception was tolerable given the LPW sensor locations, expected plasma environments and science operation scenarios.

To keep the spacecraft potential from being affected by the LPW voltage sweep operation, the effective conductive area tied to ground was to be at least 200 times that of the biased metal including that of the sensor. However, the conductive area in the wake of a spacecraft passing through mesothermal plasma is effectively precluded from use in the analysis. In addition, the solar array cell interconnects are positively biased and left exposed for cost reasons. Analysis showed that a ratio of 80 was acceptable, and a further examination showed that the times when the ratio was lower 
amounted to at most only a few minutes per periapse pass during the science collection phase.

The SA string switching requirement was met by the electrical power system that utilizes a digital solar array switching technique to control battery charging. As part of the overall scheme of electromagnetic interference control, the LPW operation was synchronized so that no data will be taken while the strings are being switched.

Ensuring that low frequency noise would not impact LPW performance required careful control of common mode emissions both to the power lines and to the chassis. All powered components on the spacecraft underwent testing for both conditions, and met the limits imposed by the General Environmental Verification Specification. In addition, SA generated switching noise was avoided by forcing the string switching to occur in a known time slice that the LPW could ignore. A spacecraft level radiated emissions test verified that the low frequency emissions requirement was met.

Accommodation of the LPW instrument, Fig. 9, required several design solutions. First, the requirement to keep the LPW sensor at least $5 \mathrm{~m}$ from the spacecraft lead to the selection of a $7 \mathrm{~m}$ boom design based on a Space Sciences Laboratory (SSL) design used on multiple previously flown missions. The extra length above the requirements provided some margin against sensing spacecraft potential variations and better clearance of the solar arrays. The boom locations and angles with respect to the spacecraft ensure that at least one boom will not be in the spacecraft wake, or be shadowed during science operations.

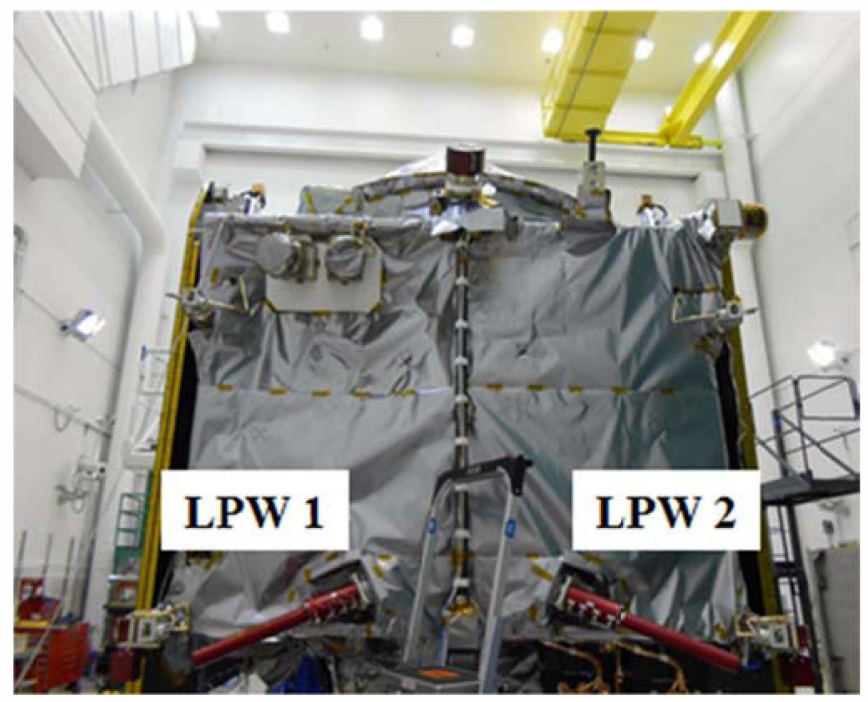

Figure 9 - LPWs mounted to the Spacecraft Lower Deck with Red Tag Covers installed

Although not a requirement, the sensor whip was changed from a hinged arrangement to a fixed configuration between instrument Preliminary Design Review (PDR) and Critical Design Review, reference Fig. 10. The fixed configuration fits within the launch vehicle fairing static and dynamic envelopes, Fig. 11. Vibration tests verified that the whip would survive launch and their success lead to the adoption of the fixed configuration which reduced both risk and cost.

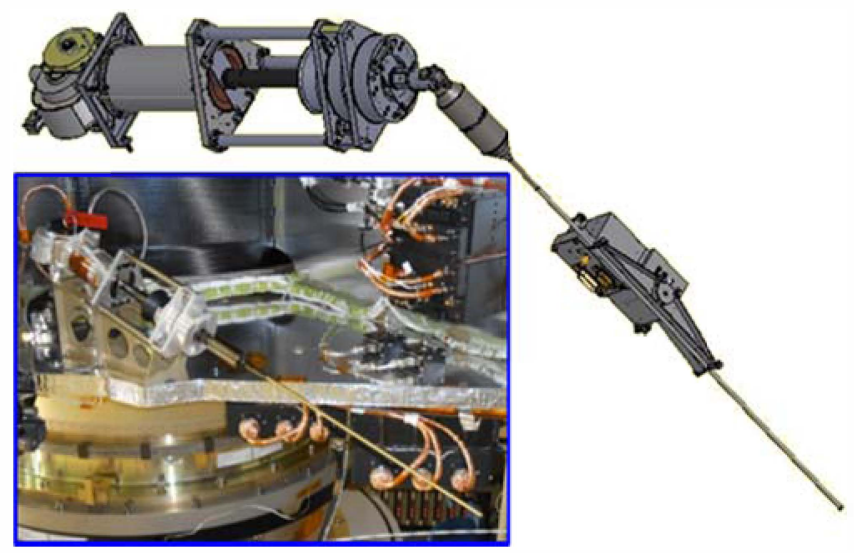

Figure 10 - LPW hinged PDR Design and Final Fixed Design installed on the Spacecraft

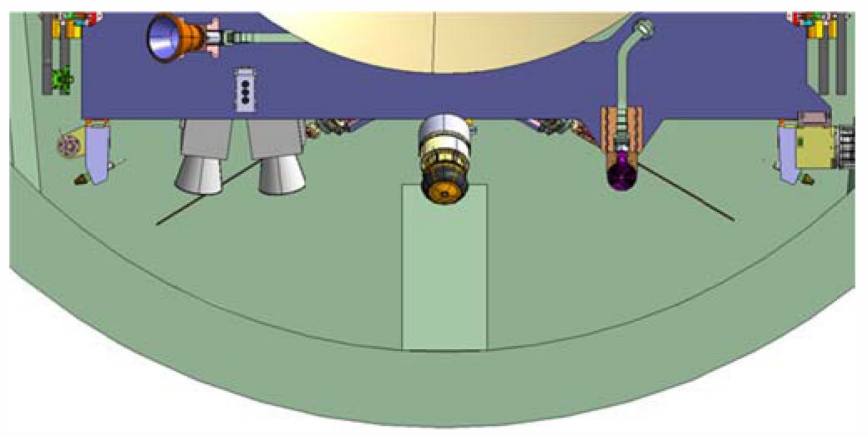

Figure 11 - LPW "Whips" within Fairing Envelope

The requirement for minimum frequency is driven by spacecraft control modes. Meeting the minimum frequency in the deployed configuration was a simple matter of choosing the correct stator wall thickness. The requirement for removal was solved by a threaded fitting at the base of the sensor whip and protected the sensor from damage during Assembly, Test and Launch Operations (ATLO).

\section{Test Program}

The MAVEN test program was very much like any other spacecraft, but involved some very mission specific tests. The following sections will cover component/instrument level tests that were critical to verifying certain aspects of instrument performance/interfaces and system level tests that addressed the magnetic requirements, deployments, and the first time observatory vibration test at the Lockheed Martin (LM) Waterton Facility.

\section{Instrument Level Tests}

Magnetics - The MAVEN approach to magnetic cleanliness was to build detailed static and dynamic magnetic models based on magnetic dipole moment measurements of all 
spacecraft components including the instruments. Given the distance between the moments and the magnetometers, measuring to higher orders (e.g., octupole) provided no benefit. These models were then validated by a set of systems/spacecraft level tests to be described later. The solar arrays were modeled separately as an assembly of field generating line segments representing the panel solar cell layouts and backwiring.

The magnetic dipole of the RS Package was measured by the Laboratory for Atmospheric and Space Physics (LASP) at the Ball Aerospace facility in Boulder, and the dipoles of the PFP were measured by SSL at the University of California (UC) Berkeley. All the other components mounted on the spacecraft, including NGIMS and the Electra transceiver, were characterized at the LM Magnetics Laboratory located at their Waterton facility, Fig. 12. All components were measured in their powered off case, and also in their powered on state as required. Structural items, while non-magnetic by design, were surveyed at a distance of 1 inch with a Gauss meter to verify their expected state.

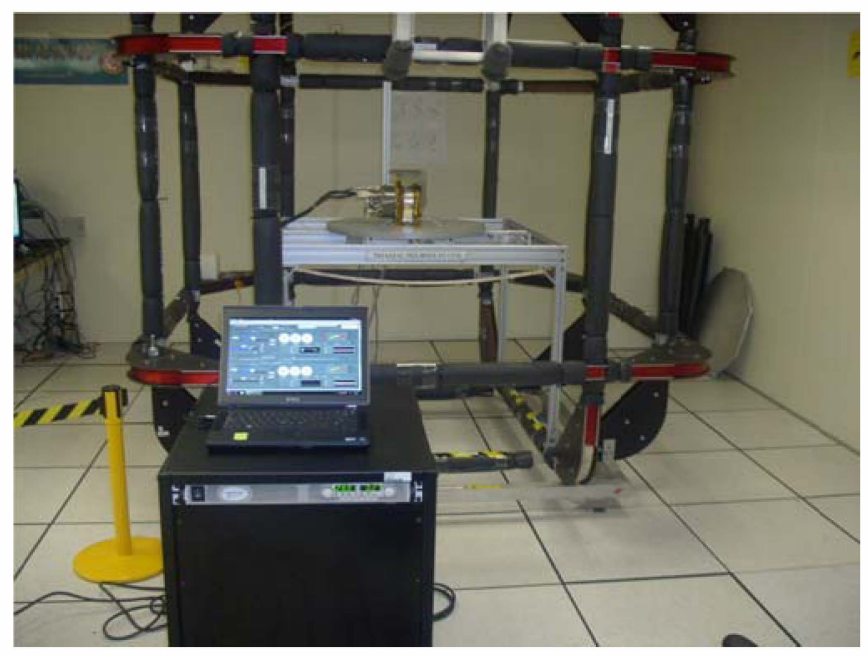

Figure 12 - APP Gimbal Motors in Magnetic Test Facility

A number of components (e.g., latch valves, traveling wave tube amplifiers) contained large ferromagnets and required magnetic compensation. Strategic placement of small rareEarth magnets reduced the dipole of each compensated component by roughly $90 \%$. During component testing, an unexpectedly large dipole was found in the Electra transceiver that was compensated for and resulted in a very small impact to the modeled field at the magnetometers.

Dynamic components (e.g., reaction wheel assemblies, gimbal motors, batteries) do not lend themselves to static compensation as above. These elements were shielded with a high-permeability foil that reduced the observed fields by $50 \%$ to $60 \%$ with only modest impact to the mass budget. The reductions were sufficient to meet the dynamic cleanliness requirement.

Measurements of the solar array magnetic properties were performed on qualification articles that contained solar array strings wired in the flight configuration. Field generation was accomplished by injecting a reverse current into selected strings. The fields were then measured over a transect across the strings. Since these measurements were performed in a magnetically noisy environment, a synchronous detection software algorithm was used to remove the noise.

A conscious effort was made during ATLO to ensure that all tools and materials brought into the vicinity of the spacecraft were demagnetized. This effort required development of procedures and training for all personnel, including the instrument teams.

LPW Tests - True LPW instrument performance can only be verified in the presence of a plasma field in space. For MAVEN several tests were performed at the LPW instrument level to ensure proper performance once at Mars. First, the sensor pre-amplifier and whip were calibrated with their electronics and then underwent thermal vacuum testing. The booms underwent deployment testing and then thermal vacuum testing where the booms were run through deployment sequences under hot and cold vacuum conditions. Measurements were made after each deployment for run out, hysteresis and sensor resonance with a dummy sensor. The booms' magnetic properties were verified prior to Thermal Vacuum (TVAC) testing.

\section{System Level Tests}

Magnetics - Three different spacecraft level tests were used to verify the models developed using data from the component/instrument level tests: swing/rotation tests, powered tests, and the APP motion tests. The swing/rotation tests were used to verify the static dipole of the spacecraft and identify any inadvertently magnetized items. The powered test verified the dynamic field estimates of individual components, and identified any inadvertent current loops. The APP motion test acted as a phasing test for the fields generated by currents, rotating elements and static dipole motions. Further details are provided below.

The difficulty of measuring weak magnetic fields in a noisy environment can be mitigated by taking advantage of the field $1 / \mathrm{r}^{3}$ dependence and placing the test magnetic sensors closer to the spacecraft. In the case of MAVEN a further step had to be taken to remove the facility noise. This required hoisting the spacecraft on a non-magnetic sling (Fig. 13), pulling the spacecraft slightly off vertical, and allowing it to execute a few pendulum motions with respect to the test magnetometers. Software can then remove the noise by locking in on the motion. During this test a number of non-magnetic items (e.g., solar arrays) were not installed. After the swing tests were executed on the spacecraft $X$ and $Y$ axes, the spacecraft was held stationary and then rotated about the $\mathrm{Z}$ (hoist) axis in both the clockwise and counter clockwise directions. The swing tests measured the total DC dipole moment and its offset along the $Z$ (vertical) axis. The measurements taken while rotating about the $\mathrm{Z}$-axis gave 
the offset of the dipole with respect to the center of the spacecraft. In addition, the rotations permitted the identification of inadvertently magnetized items. None were found.

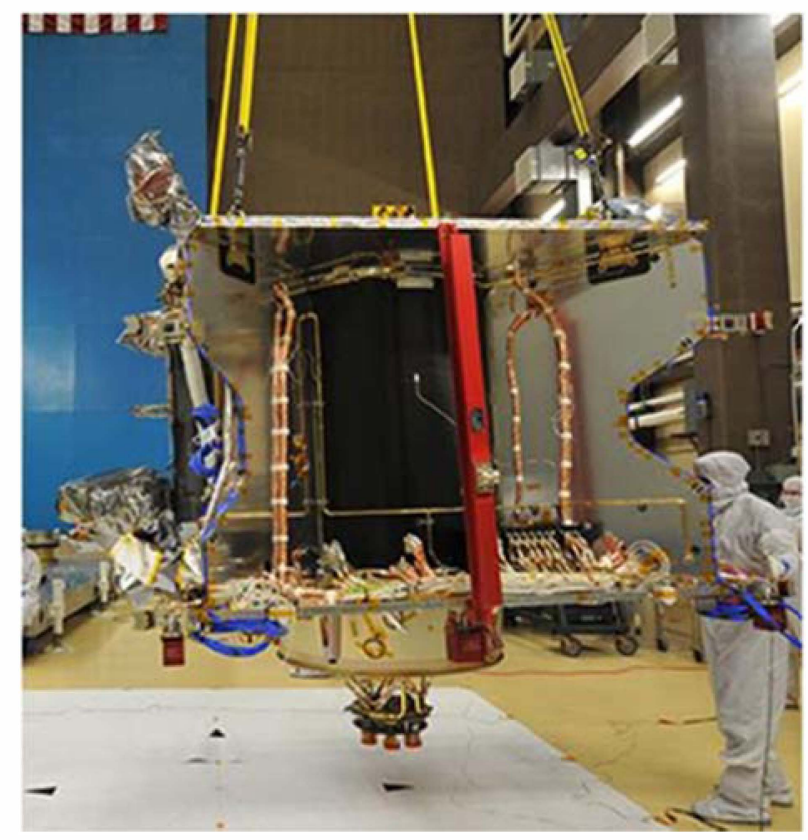

Figure 13 - Spacecraft Suspended for Magnetic Swing / Rotation Test

The results of these tests showed good correlation with the static model prediction with both magnetometers showing static field magnitudes $<1 \mathrm{nT}$. During these tests higher than expected fields were observed on the Y-axis swing that were traced to the magnetic moments induced in the high permeability shields by Earth's field. Component testing was done in a zero field environment using a Helmholtz coil. Once this effect was taken into account, theory matched observation to within a small offset.

The powered tests saw the turn on/off of all components including heaters. Unlike the swing/rotation tests, MAVEN was in a near flight configuration. The same Earth field impact to high permeability material was observed, and once accounted for, the models correlated with observation. Two deviations were discovered, one associated with the battery shielding configuration and the other to fuel tank heater wiring. Both were corrected and performance verified by retest.

The APP motion tests were conducted in the later stages of ATLO using test magnetometers provided by the GSFC magnetometer team. Both the inner and outer gimbals were exercised and showed good correlation with model predictions.

Final validation of the entire magnetic test and analysis is performed on-orbit. Twice during the cruise phase, the spacecraft will be commanded through a series of maneuvers to sweep the magnetometers through the ambient magnetic field in order to measure the background spacecraft magnetic emissions. The first time occurs shortly after entering the cruise phase as an initial checkout. The second happens closer to Mars to obtain more representative solar wind measurements.

$L P W$ Tests - Several tests were performed at the spacecraft level to verify that LPW would survive launch and operate at Mars. First, LPW sensors (minus protective covers) had to survive both spacecraft acoustics and sine vibe testing. The next test was the spacecraft radiated emissions test showing that the emissions below $4 \mathrm{MHz}$ met requirements and finally a spacecraft self compatibility test was run to verify that the PFP, not just the LPW circuitry, would operate with all the other spacecraft components.

Vibration - Sine vibration testing had not been performed on previous interplanetary LM spacecraft, but it is a cornerstone of almost all GSFC mission environmental testing. To this end, the sine vibration test of the MAVEN spacecraft was a key element of the system verification plan and was performed at LM's new vibration test facility, Fig. 14. This test subjects the spacecraft to a sinusoidal vibration base input from 10-60 Hz derived from coupled load cycle results (1.25 $\mathrm{x}$ limit) and represents a dynamic workmanship/proto-flight test of the MAVEN flight hardware. The overall objectives of this test were:

- Verify stability and performance of powered-on components during and after each axis test.

- Verify mechanical integrity of spacecraft components following each axis test (visual inspection/coin tap and low-level sine sweep).

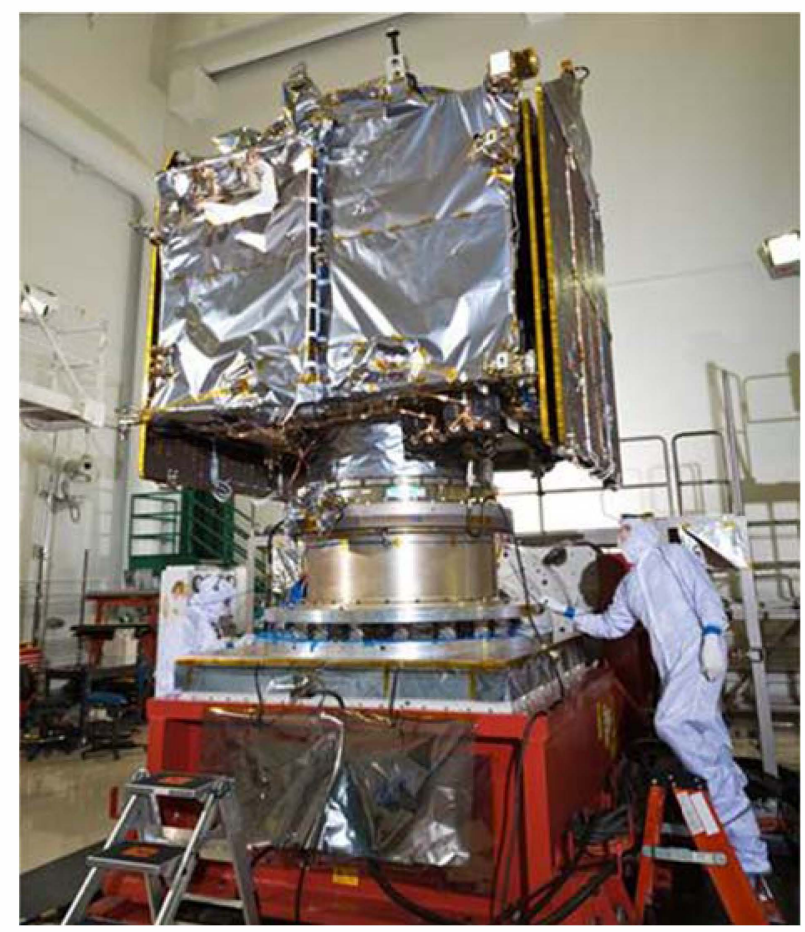

Figure 14 - Spacecraft Mounted to the Vibration Table 
The spacecraft was in the launch powered configuration during the vibration testing with most observatory hardware installed. Mass simulators were used in place of flight hardware that was not available at the time of testing. A combination of force sensors and accelerometers was used to control the test input levels as well as collect response data for all critical subsystems. Bare table runs were performed prior to installation of the spacecraft on the fixture to verify control functions and correct operations. Spacecraft self-tests were run between axes to verify all spacecraft functions.

The testing verified the following requirements: components and instruments with a fundamental resonance below $100 \mathrm{~Hz}$ was verified to the Coupled Loads Analysis (CLA) loads or to the Mass Acceleration Curve (MAC) loads, the structures subsystem with all components installed suffered no structural failure or detrimental deformation from exposure to Quasi-Sinusoidal Vibration loads defined in the Environmental Requirements Document, and the spacecraft was shown to be compatible with the dynamic flight environment shown in the Launch Vehicle ICD.

Deployments - Deployment testing was performed on the APP, SWEA boom, and Solar Arrays. Tests were conducted both at LM and KSC to verify performance both pre- and post-ship. No anomalies were encountered. The overall objectives of this testing were:

- Expose the spacecraft to the flight shock environments.

- Verify command and operation of the separation event following exposure to launch environment.

- Verify proper release and deployment with no interference with other spacecraft hardware.

- Verify stability and performance of powered-on components during the shock/deployment event.

- Verify mechanical and electrical integrity of spacecraft following shock exposure.

- Measure component shock environment and compare to component qualification environments.

The APP Deployment Test, Fig. 15, subjected the vehicle and instruments mounted to the APP to the flight level Retention and Release (R\&R) hardware separation shock environment and represents dynamic workmanship tests of the spacecraft. The results of the R\&R shock response was used to verify the MAVEN component shock environments as well as augment the LM Shock Response Database.

Multiple requirements were verified during the test including: Two-Axis Mechanism Electronics (TAME) 1553 and power interfaces, separation nut functions, and associated shock environment.

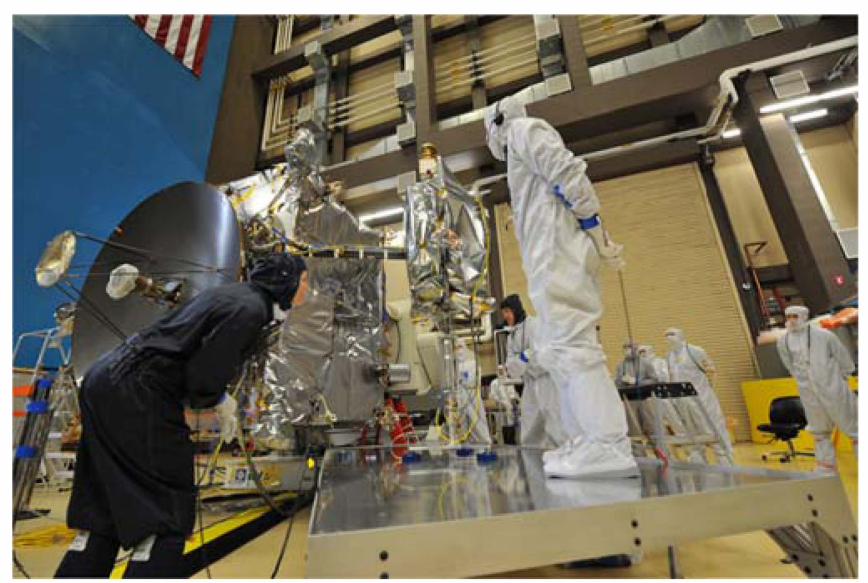

Figure 15 - APP Deployment Test

As with the APP deployment, the Solar Array Deployment test, Fig. 16, also subjected the vehicle to the flight level R\&R separation shock environment and represents dynamic workmanship tests of the spacecraft.

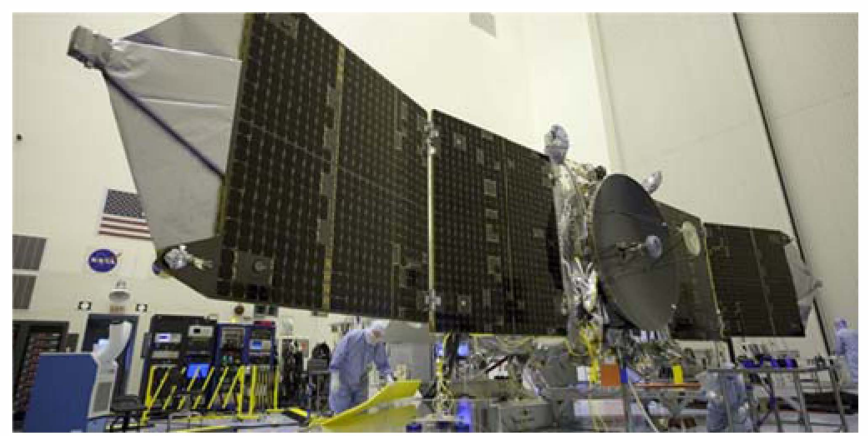

Figure 16 - Solar Array Post-Deployment

Multiple requirements were verified during the test including: electrical power system, pyrotechnic electronics, and separation nut functions. All met their performance requirements after exposure to the pyrotechnic shock environment.

Finally, the SWEA Boom Deployment Test subjected the vehicle and the SWEA instrument to the flight level R\&R separation shock.

The only critical requirements verified during this test were: SWEA nominal function after exposure to the pyrotechnic shock environment (Note: this couldn't be verified until system level TVAC due to restrictions on high powered operations in ambient conditions), pyro critical functions and separation nut functions.

\section{Conclusions}

The success of the instrument integration on MAVEN rested solely on the people involved and their commitment to defining interfaces early in the project, refining those interfaces as required, and a solid test program. The development of ICDs as early as possible in the project allowed both the spacecraft and instrument providers to 
fully understand, and more importantly agree on, the requirements that they needed to design to. One example that demonstrates the flexibility and relationship between both parties later in the project lifecycle was the change from having the spacecraft team to having the MAG team provide the mounting bracket to the SA. This change was not the result of not having the correct requirements, but rather due to the depth of requirements understanding on the MAG team as well as their experience in design/fabrication to meet those requirements as demonstrated on other missions. The test program, while only selectively covered in this paper, was thorough in all aspects of verification, within the limits imposed, and at all levels of assembly. Testing was performed at the piece part level as applicable all the way up to the observatory level. The success of this planning and testing will ultimately be measured by MAVEN's success at Mars, but the groundwork has been laid to enable that success.

\section{References}

${ }^{1}$ MAVEN Magnetometer Investigation (MAG): MAG Functional Requirements Document, May 25, 2011, MAVEN-MAG-RQMT-0061.

${ }^{2}$ MAVEN Concept Study Report, NASA GSFC, May 2008.

\section{BIOGRAPHY}

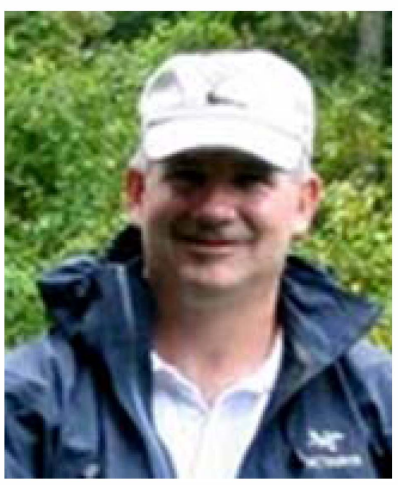

Nicholas Jedrich began his career in 1986 with Rockwell supporting the Mission Operations Directorate at the Johnson Space Center. In 1994 he joined GSFC in support of the Hubble Flight Servicing Project, where he spent 13 years working in hardware systems engineering. Nick was selected as the Express
Logistics Carrier (ELC) Deputy Project Manager in September 2007. He joined the MAVEN Team in March of 2010 as the Instrument Systems Manager, and transitioned to the Observatory Manager in the summer of 2012. He is currently the Deputy Project Manager for Payload for the Transiting Exoplanet Survey Satellite (TESS) mission.

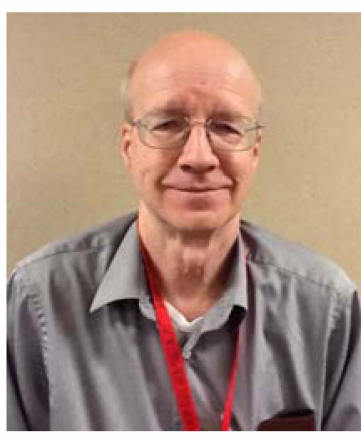

$\begin{array}{lll}\text { Regan Howard began } & \text { his } \\ \text { career in } 1976 \text { at } \\ \text { Westinghouse } & & \text { in }\end{array}$ manufacturing software and integrated circuit process development. In 1984, he joined Fairchild Space Company (now Orbital Sciences) to begin his long involvement in space system development, including architecture development for the single board computers on the SAMPEX and HST missions, and spacecraft concept development for the Dawn Discovery and ICON SMEX missions. In June 2009, he joined the MAVEN systems engineering team as the environmental requirements and test engineer. As part of this activity he managed the effort to make the spacecraft magnetically, and electrostatically clean. He is currently part of the MAVEN post-launch systems engineering support group, as well as an EMI/EMC consultant for other several GSFC missions.

\section{ACKNOWLEDGEMENTS}

The authors wish to acknowledge the MAVEN Team for their contributions to this paper. 